\title{
Linx
}

Revue des linguistes de l'université Paris X Nanterre

$56 \mid 2007$

Linguistique des genres

\section{Le modèle du Cercle de Bakhtine à l'épreuve des genres de la presse}

\section{Sophie Moirand}

\section{(2) OpenEdition}

\section{Journals}

Édition électronique

URL : http://journals.openedition.org/linx/366

DOI : $10.4000 /$ linx.366

ISSN : 2118-9692

\section{Éditeur}

Presses universitaires de Paris Nanterre

\section{Édition imprimée}

Date de publication : 1 juin 2007

Pagination : 91-108

ISSN : 0246-8743

\section{Référence électronique}

Sophie Moirand, « Le modèle du Cercle de Bakhtine à l'épreuve des genres de la presse », Linx [En

ligne], 56 | 2007, mis en ligne le 21 février 2011, consulté le 20 avril 2019. URL : http:// journals.openedition.org/linx/366 ; DOI : 10.4000/linx.366 


\title{
Le modèle du Cercle de Bakhtine à l'épreuve des genres de la presse
}

\author{
Sophie Moirand \\ Université Sorbonne nouvelle - Paris 3, Cediscor - Syled
}

La lecture de deux textes de Bakhtine traduits en français en 1984 (Bakhtine 1952-53 et 1959-61) rapportée à celles des textes de Voloshinov déjà traduits dans Todorov 81 (Voloshinov 1926 et 1930) m'ont conduite à proposer un modèle dialogique pour analyser les genres de la presse écrite (Moirand 2007a pour une synthèse). Je reprendrai brièvement ici ce que j'ai déjà largement expliqué ailleurs (Moirand 2003b, pour une approche du "genre», et 2005b, 2006a, pour celle du "dialogisme »), à savoir qu'il ne s'agit pas d'user de la notion de dialogisme comme d'une catégorie complémentaire de celles des théories énonciatives classiques (le cadre indiciel et le cadre pragmatique). Il s'agit plutôt de considérer que la réflexion sur les genres de 1952-53 demeure indissociable de la théorie de l'énoncé et de la conception de la situation proposée par Bakhtine et/ou Voloshinov (désormais $B / V$ ) ${ }^{1}$ ainsi que d'une linguistique et d'une translinguistique qui ne sont pas deux disciplines disjointes mais complémentaires : le discours, objet de la translinguistique, est fondamentalement lié aux formes de la langue et au «tout» que constitue l'énoncé (ou le genre ?) ; mais « la langue vit et évolue historiquement dans la communication verbale concrète, non dans le

\footnotetext{
${ }^{1}$ Je ne discuterai pas la paternité des textes du Cercle de Bakhtine, terme que j'emprunte à Todorov pour ne pas me prononcer sur cette question à laquelle je ne peux répondre (voir ici même la mise au point de Jean-Paul Bronckart.). C'est pourquoi la mention $\mathrm{B} / \mathrm{V}$ me parait dans certains cas opportune.
} 
Sophie Moirand

système linguistique abstrait des formes de la langue, non plus que dans le psychisme individuel des locuteurs » (B/V, 1977, p. 137) $)^{2}$, donc dans ses usages sociaux.

Cela veut dire que ce n'est pas la notion de dialogisme «décontextualisée » de l'environnement théorique construit pas le Cercle de Bakhtine au fil des articles signés Voloshinov et Bakhtine que l'on emprunte, mais, avec elle, c'est toute une conception du langage (du sens et du discours), tel qu'il est ancré dans l'histoire et la société, auquel on se réfère. Or ce que disent $\mathrm{B} / \mathrm{V}$ de l'articulation de l'objet de la linguistique et de la translinguistique m'a semblé d'emblée infiniment proche d'une analyse du discours dite «française » (ADF), qui prend appui sur les formes de la langue pour traquer l'interdiscours qui s'y blottit, analyse théorisée par Pêcheux (1975 et dans Maldidier 1990), pour qui, d'après Maldidier (1993, p. 89), « le sujet n’est pas la source du sens» car «le sens se forme dans l'histoire à travers le travail de la mémoire, l'incessante reprise du déjà-dit», et donc du discours, devrait-on ajouter... Ainsi les concepts principaux de l'ADF, ceux d'interdiscours et d'intradiscours, de pré-construit et de mémoire discursive, voire de formation discursive, peuvent-ils être repensés à la lumière des conceptions du langage de B/V (Moirand 2000), qui permettent de recentrer l'analyse sur des objets d'études que l'ADF avait occultés ou oubliés: la notion de genre discursif ainsi que "l'hétérogénéité "extrême" des genres du discours » (Bakhtine, 1984, p. 267).

Comment le modèle $\mathrm{B} / \mathrm{V}$ permet-il de porter un autre regard sur les genres de la presse, et de construire ainsi un objet de recherche différent des conceptions communicationnelles des médias? Comment une analyse de données empiriques empruntées à la presse quotidienne permet-elle de concevoir un modèle de description du genre en tant que lieu de rencontre de discours empruntés à des communautés langagières appartenant à des mondes sociaux différents? Comment les interactions discursives qui composent les genres, chacun différemment, permettent-elles de mettre au jour des différences entre genres, en particulier à travers les usages différents qui sont faits de l'intertexte ou de l'interdiscours? Comment enfin repérer les différentes formes d'appel à la mémoire, et donc à l'histoire, qui semblent ainsi inscrites dans les mots, les formulations, les constructions, les énoncés qui se glissent au fil des textes de presse ?... Telles sont les questions que l'on s'est posées au fur et à mesure des analyses effectuées sur la presse quotidienne.

\section{Des genres du discours aux genres de la presse}

Si, comme le dit Bakhtine, chaque sphère de l'activité humaine a son propre répertoire de genres, avec ses normes de fonctionnement, il est normal qu'au fur et à mesure du surgissement de nouvelles activités ou de l'évolution de certaines pratiques professionnelles, liées souvent à l'évolution des supports (dans les médias, avec l'internet, avec l'apparition des «gratuits »...), on assiste à une reconstitution des répertoires. C'est ainsi qu'une communauté langagière (notion que l'on substitue à celle de sphère...) dispose d'un répertoire générique non pas fixe mais évolutif, et c'est le cas des communautés langagières professionnelles qui appartiennent au monde social

2 Dans les citations et les exemples, les italiques sont toujours celles du texte original. 
des médias ${ }^{3}$. Comment rendre compte de la diversité, de l'évolution et de la complémentarité des genres de ces communautés, genres qui sont essentiellement constitués de discours médiateurs?

\subsection{Bref retour sur le Cercle de Bakbtine}

Ce que le texte de Bakhtine sur «les genres du discours» apporte, c'est pour l'essentiel une critique à la conception de Saussure, d'une part que la parole serait individuelle, d'autre part qu'il existe, au-delà des structures de la langue, une structuration globale du «tout » que constitue l'énoncé : «Saussure ignore le fait qu'en dehors des formes de la langue existent aussi les formes de combinaison de ces formes, c'est-à-dire qu'il ignore les genres discursifs » (Bakhtine 52-53, traduction dans Todorov 1981, p. 90), et si « tout énoncé pris isolément est, bien entendu, individuel», "chaque sphère d'utilisation de la langue élabore ses types relativement stables d'énoncés, et c'est ce que nous appelons les genres du discours. (Bakhtine 1984, p. 265).

Pour bien comprendre cependant cette conception du genre, il faut la rapporter à la théorie de l'énoncé (Todorov, 1981, chap. 4), et en particulier aux deux textes de Voloshinov signalés en introduction, comme je l'ai proposé dans Moirand 2003a. Il me semblait en effet que les catégories présentes dans la définition maintes fois citée qui figure en première page du texte de 1952-53 (contenu thématique, style, construction compositionnelle) débouchaient souvent sur une conception du texte plutôt que du discours... En revanche, on trouve dans les textes antérieurs de Volochinov à la fois une conception originale de la situation, avec sa composante évaluative ${ }^{4}$, qui, loin d'être une cause extérieure de l'énoncé, constitue un élément de sa configuration sémantique, ainsi qu'un modèle top down de détermination socio-historique des énoncés (voir Todorov, 1981, p. 190-192) :

- [Le] contexte extra-verbal de l'énoncé se décompose en trois aspects: 1) l'horizon spatial commun aux locuteurs [...] 2) la connaissance et la comprébension de la situation, également commune aux deux locuteurs et, enfin, 3) l'évaluation - commune là encore - qu'ils font de cette situation.

\footnotetext{
${ }^{3}$ Dans les analyses réalisées sur la presse écrite, on distingue "des "mondes sociaux" (le monde politique, le monde scientifique, le monde médiatique, le monde de l'industrie et du commerce, le monde associatif....), qui sont eux-mêmes constitués de "communautés langagières" diversifiées, fonctionnant comme des institutions régulant des pratiques langagières auxquelles se soumettent les membres $d u$ groupe et qui s'actualisent dans des genres discursifs, voire des dires, propres à la communauté, que ces normes soient implicites ou explicitées » (Moirand 2007a, p. 94-95).

${ }^{4}$ L'évaluation au sens de B/V pourrait être rapportée aujourd'hui aux conceptions qui postulent un traitement cognitif de construction/reconstruction du (ou des) contexte(s) intervenant antérieurement et simultanément dans la production du discours, et qui reposerait sur le répertoire générique des interactants : voir à ce sujet « l'interface cognitive » de T. van Dijk qui s'appuie sur les représentations sociocognitives que se font les interactants de la situation (Micheli 2006) et non pas sur la situation "concrète ", ainsi que la notion de prédiscours proposée par M.-A. Paveau 2006 (Moirand 2007b).
} 
Sophie Moirand

[...] la situation extra-verbale n'est en aucune façon la cause extérieure de l'énoncé, elle n'agit pas sur lui de l'extérieur comme une force mécanique. Non, la situation s'intègre à l'énoncé comme un élément indispensable à sa constitution sémantique.

[Voloshinov, 1926, traduit dans Todorov 1981, p. 190-191]

- [...] il serait vain de chercher à résoudre le problème de la structure des énoncés dont est faite la communication, sans tenir compte des conditions sociales réelles c'est-à-dire de la situation - qui suscite de tels énoncés. [...] : l'essence véritable $d u$ langage, c'est l'événement social qui consiste en une interaction verbale, et se trouve concrétisé en un ou plusieurs énoncés.

Quant à la modification des formes du langage, comment se réalise-t-elle ? de quoi dépend-elle ? selon quel ordre se déroule-t-elle ? Les données du précédent article nous permettent d'élaborer un schéma qui les synthétise et se trouve, justement, répondre aux questions qu'on vient de poser:

1) Organisation économique de la société.

2) Rapport de communication sociale.

3) Interaction verbale.

4) Enoncés.

5) Formes grammaticales du langage.

Ce schéma nous servira de fil directeur dans l'étude de cette unité concrète, qui relève de la parole et que nous appellerons énoncé. [...]

[B/V. ,1930, dans Todorov 1981 : 288-289].

Ainsi si, dès 1926, il apparaît une relation entre l'interne (la structure de l'énoncé) et ses extérieurs (le contexte extra-verbal), l'article de 1930 élargit le contexte à l'organisation de la société. Se référant alors à l'ouvrage publié l'année précédente (traduit en français en 1977 sous le titre Le marxisme et la philosophie du langage), la relation avec le genre est explicitement établie dans une longue citation concernant les genres quotidiens (Voloshinov, dans Todorov 1981, p. 291-291).

On a retenu alors de ces différents écrits que si le genre était pensé comme un tout constitué de composants internes, il inscrivait également dans sa structuration sémantique les constituants de la situation, telle que la perçoivent et l'évaluent les interlocuteurs, dans ses déterminations extérieures d'ordres spatial (la sphère d'activité) et temporel ( « au niveau de la famille, de la nation, de la classe sociale, des jours, des années et des époques entières»). Et comme «la particularité des énoncés quotidiens », c'est d'être « reliés par des milliers de fils au contexte vécu extra-verbal » à tel point que si «on les détache de ce contexte, ils perdent la quasi-totalité de leur sens » (Volochinov 1926, dans Todorov, p. 190-192), l'hypothèse que l'on fait, c'est que l'on peut accéder au moins partiellement à ces extérieurs grâce au repérage des différents formes de dialogisme, catégorie «qui peut aller du dialogue ouvert à l'allusion la plus discrète » (selon Todorov commentant le texte de Bakhtine de 1959-61 : Le problème du texte). C'est ainsi que, dès les premières lectures effectuées au début des années 1980 (B/V 1977, Todorov et Volochinov 1981), j’avais déjà décidé de mettre le dialogisme à l'épreuve de données empiriques à des fins de classification des unités discursives rencontrées dans une revue spécialisée (Moirand 1988) avant de le faire pour les genres de la presse quotidienne. 


\subsection{De l'objet d'étude (la presse) à l'objet de recherche}

La notion de «genres de la presse écrite » semble faire désormais l'objet d'un consensus, qu'on les désigne par les étiquettes en vigueur dans la profession et les manuels de journalisme, ou qu'on essaie, en les prenant un par un comme objet d'étude, de dégager des caractéristiques fondées sur des «critères minimaux » qui permettraient de les différencier: sémantique, énonciatif, pragmatique, compositionnel, stylistique, longueur... (voir Adam 1997). Il reste que de nombreux travaux, en particulier en sciences du langage, mais également en sciences de l'information, portent sur un seul genre, et en particulier sur les genres les mieux «stabilisés » (le reportage, le fait divers, l'éditorial, l'interview, la critique d'objets culturels, etc.), voire sur une catégorie linguistique, pragmatique ou textuelle (les temps, le discours rapporté, la désignation, le récit, l'inscription de la personne, la modalité appréciative, etc.) ${ }^{5}$.

Mais lorsqu'on s'interroge sur les genres tels qu'ils se répartissent au fil des rubriques ou du déroulé du journal quotidien, ou tels qu'ils se distribuent sur l'aire de la page ou de la double page, on se trouve face à ce que j'appelle des unités discursives empiriques qui relèvent "visiblement» de genres différents mais dont les classifications proposées par la communauté langagière professionnelle manifestent " une hétérogénéité » et « un flou définitionnel décourageant » (Adam 1997, p. 4).

Pour venir à bout de cette hétérogénéité multiforme (textuelle, iconique, énonciative) qui saute aux yeux de l'observateur et pour éviter le risque de décontextualisation que court l'étude d'un seul genre (qui présente l'avantage de l'homogénéité du corpus) ou même d'une seule page ou double page ${ }^{6}$, on a fait un premier choix : celui de prendre comme objet d'étude un «moment discursif », lorsqu'un fait donne lieu à une intense production discursive dans les médias et à une diversité de genres discursifs dans la presse qui se distribuent sur l'aire de la page, au fil du journal et des numéros. Faisant alors l'hypothèse que des genres différents interviennent dans la construction discursive de l'événement en traitant chacun d'une de ces facettes, et que les genres de la presse se différencient par l'utilisation différente qu'ils font des textes des autres, donc par les traces d'intertextualité ou d'interdiscursivité qui les traversent, l'objet de recherche consiste alors à mettre au jour les types de texture énonciative des différents genres rencontrés: les places énonciatives que le discours construit et en particulier les représentations qu'il donne du discours des autres.

Ainsi, à partir d'une partition «sommaire » entre les textes dits à énonciation plutôt objectivisée (textes d'information, brèves, enquêtes, chronologies, glossaires, tableaux, graphiques...) et les textes dits à énonciation plutôt subjectivisée (commentaires, éditoriaux, dessins de presse, analyses, tribunes...), on a pu mettre au jour différents

\footnotetext{
${ }^{5}$ On peut ainsi consulter, par exemple, les numéros 13 et 22 de la revue SEMEN, le numéro 94 de la revue Pratiques, les numéros 34 et 134 de la revue Le français aujourd'bui, ainsi que l'ouvrage de E.U. Grosse et E. Seibold 1996.

${ }^{6}$ Certains quotidiens réunissent les genres du commentaire sur une même page ; et si une page ou une double page constituée de genres hétérogènes réunis autour d'un thème ou d'un événement permet une contextualisation spatiale partielle, elle fait limpasse sur la dimension temporelle, indispensable à la saisie du traitement médiatique d'un événement.
} 
Sophie Moirand

aspects du dialogisme, tels qu'ils s'inscrivent dans la matérialité verbale. La présence sur une même page d'un article d'information scientifique dont l'essentiel des données était repris de la revue Nature et d'un article sur les relations entre science et politique, articles rédigés par le même journaliste à propos d'un même moment discursif (la crise dite de la vache folle), a par ailleurs confirmé l'hypothèse que l'utilisation de l'intertexte (qu'on percevait dès le premier regard : citations en italiques, références situées dans le premier ; allusions à du discours autre non situé et à des actes de langage de groupes d'énonciateurs anonymes dans l'autre) n'était pas due au scripteur ni au thème ni au journal mais à des contraintes génériques (Moirand 2001).

On a alors défini, à partir d'un corpus constitué de moments discursifs ayant trait à l'alimentation, à la santé ou à l'environnement, différentes formes d'actualisation du dialogisme, que l'on a rapportées aux différentes classes d'unités empiriques rencontrées au fil des pages et des numéros de plusieurs quotidiens (on trouvera de nombreux exemples dans Moirand 2001, 2005b, 2006a et 2007a) :

- Les genres de l'information se caractérisent par une dimension intertextuelle explicitement «montrée ». Les dires rapportés, nominalement attribués à leurs auteurs (nom, spécialité, lieu d'exercice, lieu de parole, genre d'origine...), ici des spécialistes à qui on passe la parole, donnent une valeur d'authenticité aux informations données dans l'article. Ce qui compte, c'est peut-être moins ce qui est dit que le fait que ce soit dit par des membres de la communauté «experte », pour justifier les explications proposées en réponse aux questions que les destinataires pourraient poser. L'intertexte est à la fois montré et «situé ».

- Les genres à coloration didactique (glossaires, encadrés explicatifs, etc.) présentent le plus souvent une texture particulière : un dialogisme qui se caractérise à la fois par l'effacement des marques d'emprunt à du discours autre (dialogisme intertextuel masqué) et par l'inscription des dires qu'on anticipe chez les destinataires, par exemple dans les titres, les intertitres qui accompagnent le genre ou et qui entrent dans sa composition. Cette dimension interactionnelle du dialogisme, représentative de genres de l'explication, est donc plus ou moins présente dans la matérialité textuelle.

- Les genres du commentaire, qui fonctionnent majoritairement à coup d'allusions plutôt que de citations, montrent souvent différentes traces de ce qu'on a appelé une hétérogénéité suggérée. Or ce dialogisme-là constitue le troisième degré défini par Bakhtine, celui où « le discours d'autrui n'est attesté par aucun indice matériel, et se trouve pourtant évoqué », parce qu'il est « disponible dans la mémoire collective d'un groupe social déterminé » (Todorov 1981, p. 113).

Il est alors apparu qu'il fallait affiner la méthode, et cela à partir de deux observations essentielles :

- À côté de genres de l'information qui se caractérisent par un intertexte montré «monologal» (la voix d'une communauté scientifique homogène), conforme au modèle traditionnel de la vulgarisation scientifique (le média joue son rôle d'intermédiaire entre le discours de la science et celui du public), on était frappé, dans le cas des événements scientifiques à caractère politique récents, de la diversité des discours autres auxquels les genres de l'information faisaient appel et par conséquent de la diversité des communautés langagières convoquées dans une même unité discursive. Or, ce dialogisme intertextuel plurilogal, qui devenait peu à peu une caractéristique des genres de l'information traitant ce type d'événements, conduisait à 
faire porter le regard non pas sur les paroles rapportées mais sur l'encadrement des segments cités afin de mettre au jour la diversité des mondes sociaux convoqués ainsi que les rencontres discursives (voire les controverses) que le genre met en scène, souvent des interactions entre des énonciateurs qui n'ont pas forcément l'occasion de se croiser dans l'espace social...

- Face à l'hétérogénéité suggérée de certains titres et en particulier des textes de commentaire comme les éditoriaux ou les chroniques, qui renvoient, à coups d'allusion, aux extérieurs des énoncés, et pas seulement aux contextes de textes présents sur la page ou dans le numéro, il fallait s'appuyer sur d'autres observables, des indices de contextualisation de différents niveaux, et retrouver ainsi les différentes épaisseurs dialogiques de mots, de formulations ou de constructions qui, convoquées au fil de l'unité discursive analysée, contribuaient à son orientation pragmatique ou argumentative (Moirand 2007b).

C'est ainsi qu'une articulation s'est imposée entre le dialogisme du Cercle de Bakhtine et la notion d'interdiscours de l'ADF : dans le fil horizontal du discours (l'intratexte), des mots déjà dits, des sens déjà énoncés, des dires antérieurs, des positions énonciatives s'inscrivent subrepticement, renvoyant à des fils verticaux et à un interdiscours que l'énonciateur présent a parfois lui-même oublié, ou refoulé, donc à des domaines de mémoire et à l'histoire, à court, à moyen ou à long termes.

\section{Des caractéristiques génériques qui référent à l'histoire et à la mémoire}

Si ces premiers travaux avaient confirmé l'intérêt du dialogisme (plutôt que les catégories du discours rapporté) pour décrire les genres de la presse, ils montraient un double danger : celui de s'y référer sans le rapporter aux formes de la langue inscrites dans les unités discursives empiriques analysées; celui d'instrumentaliser la notion au risque d'en faire une catégorie descriptive supplémentaire sans lui donner "sa pleine dimension de théorisation, à la fois historique et subjective, du fait du sens ", parce que «Les mots, chez Bakhtine ne sont pas des "galets" lisses, compacts, unités d'un système linguistique abstrait, mais des matériaux "poreux", intimement pénétrés par les environnements dont ils restituent quelque chose - "allusivement" - dans le dire où ils figurent» (Authier-Revuz 2000 : 229). Il fallait d'une part, pour étudier ses différentes formes d'actualisation, la traquer dans les traces des opérations langagières de référence, de prédication et d'énonciation laissées à la surface du texte (les formes de la langue au niveau local du genre) et leur distribution, leur répartition, leur combinaison dans le fil horizontal de l'article ou du titre ou du dessin de presse (Moirand 2004a, 2005b). Il fallait d'autre part construire un nouvel objet de recherche qui s'attache à retrouver, au-delà de ce quelque chose qui constitue une trace de la mémoire $d u$ mot, les différents contextes qu'il a traversés, les trajets qu'il a déjà effectués au fil de son histoire et des situations qu'il a rencontrées.

L'analyse des constructions et des allusions qui mettent en relation des événements conduit à rechercher la fonction de ces mises en relation et à articuler le 
Sophie Moirand

sens linguistique au sens social qui apparait dans la construction discursive des événements au fil des différents genres convoqués. La notion de contextualisation est alors repensée en termes de relations intertextuelles et interdiscursives plutôt que de situation. L'objet de recherche devient de ce fait l'étude des interactions discursives que les genres construisent, et qui contribuent à mettre en relation des domaines de mémoire référant à des savoirs et à l'histoire sociale, présente, récente ou ancienne.

\subsection{Une conception dynamique du contexte}

On peut retrouver les conditions de production des moments discursifs analysés à partir des indices de contextualisation qui se distribuent au fil du l'intratexte des différents genres de la presse.

C'est une conception dynamique du contexte qui prévaut: il s'agit de repérer des observables au fur et à mesure de l'avancée du recueil des données et des analyses effectuées. Le corpus n'est donc pas clos à priori : l'hétérogénéité énonciative d'un texte, c'est-à-dire les configurations discursives qui le composent et les discours qui le traversent, incitent à retrouver l'origine des dires qui l'informent, et partant de là à élargir le corpus... jusqu'au moment où l'événement disparaît des surfaces du journal. Mais jamais complètement puisque les désignations d'un événement deviendront à leur tour des rappels de moments discursifs précédents, comme on peut le constater dans les énoncés suivants :

1. Cette affaire en rappelle fâcheusement d'autres de sinistre mémoire : la dissimulation des risques d'irradiation après la catastrophe de Tchernobyl, l'affaire du sang contaminé et celle de la vache folle $[\ldots]$

2. Le drame serait que les oiseaux migrateurs volent vers l'Afrique qui ne dispose d'aucun réseau sanitaire pour contenir le Tchernobyl aviaire.

On rejoint ici, dans l'analyse des genres de la presse, genres seconds constitutivement inscrits dans un rapport dialogique avec d'autres textes, la position de F. Rastier, pour qui l'écrit connaît une autre forme de contextualité que l'oral, celle justement qui va de texte à texte : «à l'écrit, c'est le contexte (intra- et inter-textuel) qui domine la situation - ou la supplée », et qui ajoute que "le concept d'intertexte n'est opératoire que si l'on se réfère à un corpus » (Rastier 1998, p. 106-107). La traçabilité des domaines de mémoire que l'on appelle ou qu'on rappelle est d'autre part aujourd'hui facilitée par la consultation des archives en ligne des journaux et par l'utilisation des moteurs de recherche sur l'internet (Moirand 2006b).

Plusieurs niveaux de contextualisation sont ainsi systématiquement étudiés, du contexte proche au contexte lointain, du contexte au sens étroit du terme (le cotexte) au contexte socio-historique au sens large :

- Le cotexte linguistique d'un mot, d'une formulation, d'une construction syntaxique dans un titre (que l'on peut considérer comme un genre) ou au fil d'un article : 
3. L'OGM ou la faim?

4. Un petit Mai-68 des banlieues

5. Grippe aviaire Un fléau de plus en Afrique

- Le cotexte intratextuel de l'ordre du discours avec les reprises intratextuelles au fil d'une unité discursive, qui tout en désignant par exemple un même objet du discours, inscrit à chacune des reprises un appel à la mémoire différent, la somme de ces différents «éclairages» (Grize 1992, 2005) contribuant à construire une représentation de l'objet, ici un même acteur social :

6. Cette semaine, les conservateurs seront dans la rue, contre le CPE. Car il ne faut pas se fier aux apparences. Ce sont les porteurs de banderoles qui ne veulent rien changer [...] pour qui roulent-ils au juste, ces militants de l'immobilisme?

[éditorial]

7. Bien sûr, les différences entre les violences qui ont éclaté dans certaines villes de banlieue et les prodromes des événements de mai 1968 sont écrasantes. Les révoltés ne sont pas des étudiants [...] mais des fils et petits-fils d'immigrés, relégués aux marges de la société, souvent chômeurs... Ils inspirent davantage la peur que la sympathie [...] Il y a parmi eux des voyous inquiétants [...]

Les insurgés d'aujourd'hui n'ont pas d'autre horizon que la répétition de bastons sans autre fonction que d'extérioriser leur mal-être. Cette « canaillelà ", à la différence de celle de la Commune, n’a ni mémoire ni rêves.

[Chronique]

- Le contexte de textes sur l'espace de la page ou au fil du numéro du journal, où l'on repère des échos sémantiques et formels dès les titres et de texte à texte (et jusque dans les dessins de presse, par exemple dans le Monde - voir 2007a). Ainsi, sur une même page du Monde, on peut repérer la présence d'« échos » entre les titres et les intertitres des différents articles, comme on l'a monté dans Moirand 2004a) :

8. - La France a décidé de détruire les récoltes de colza « pollué » par des OGM.

La question de l'indemnisation des agriculteurs n'est pas réglée [article d'information du journaliste scientifique du Monde]

- Les semenciers déplorent la décision du gouvernement

[autre article d'information]

- Le pouvoir britannique se montre divisé sur la question [autre article d'information du correspondant à Londres]

- Trois questions à... $\div$

Comment avez-vous réagi à cette pollution?

Avez-vous été motivé par le seul principe de précaution? 
Sophie Moirand

Comment seront indemnisés les agriculteurs?

(interview)

- Qu'un champ impur...

Séparer le bon grain de l'ivraie, sage proposition. Et, si l'on peut dire, antique principe de précaution.

(chronique de Pierre Georges)

- Le contexte des séries génériques dans lesquelles s’inscrivent les unités discursives recueillies lors d'un même moment discursif ou d'un moment discursif à un autre (les différents éditoriaux ou dessins de presse ou les glossaires, etc.). On peut ainsi voir une définition du prion accompagnée d'un conditionnel reprise quelque temps plus tard dans un encadré de même type sans conditionnel. On peut retrouver d'un éditorial à un autre des rappels à des événements ou à des dires antérieurs qu'on a déjà rencontrés dans la même série générique (par exemple, l'image du bon grain et de l'ivraie, la boitte de Pandore, la nourriture Frankenstein sont des formes récurrentes du genre éditorial et «le principe de précaution» franchit les limites de ces événements pour s'inscrire dans d'autres familles d'événements, les catastrophes naturelles comme le tsunami ou les inondations, la canicule, le terrorisme, etc.) - voir Moirand 2007a.

- Le contexte intertextuel qui permet de retrouver les textes antérieurs lorsque les propos sont situés et les données référencées. Non seulement grâce aux références qui sont données et à l'invitation qui est faite de consulter le site du journal, mais parce que des mots comme "fracture numérique», "gouvernance de l'internet», etc. appellent à revenir sur l'histoire récente, et que ce qui est dit au fil de ces lignes des relations entre les États-Unis et le reste du monde, ou l'Union européenne, rappellent cet antagonisme ancien que l'on retrouve au fil du temps et d'autres événements, ici dans les paroles rapportées d'un genre de l'information (discorde, situation de blocage, etc.) :

\section{INFORMATION SOMMET MONDIAL}

A Tunis se joue une intense bataille pour le contrôle de l'Internet L'OBJECTIF premier du Sommet mondial sur la société de l'information (SMSI) qui se tient à Tunis su 16 au 18 novembre - et dont la première phase s'est tenue à Genève, il y a deux ans (le Monde du 13 décembre 2003)- était la réduction de la "fracture numérique » entre le Nord et le Sud. Cet aspect est passé au second plan, éclipsé par la discorde, entre les Etats-Unis et le reste du monde, sur la gouvernance de l'Internet, c'est-à-dire sur le contrôle de l'architecture de nommage de la Toile. [...]

La situation de blocage est telle qu'aucune des parties ne s'attend à ce qu'une réforme concrète soit adoptée à l'issue du sommet. Le 7 novembre, à peine plus d'une semaine avant l'ouverture de celui-ci, le secrétaire d'Etat au commerce, Carlos Gutierrez, et la secrétaire d'Etat, Condoleezza Rice, adressaient au chef de la diplomatie britannique, Jack Straw, un courrier confidentiel demandant à la présidence de l'Union européenne de «reconsidérer sa position sur la gouvernance de l'Internet», rappelant que «la structure de gouvernance et la stabilité de l'Internet sont d'une importance capitale pour les Etas-Unis. 
www Sur le monde.fr : édition spéciale «Technologies »

[le Monde, 16 novembre 2005]

- Le contexte interdiscursif qui se manifeste de manière allusive dans certains mots ou certaines constructions, comme on a pu déjà l'entrevoir dans les exemples qui précèdent, au fil des évocations de dires antérieurs qui renvoient « à du discursif qui se perd dans la nuit des temps et que nous avons toujours su!» (Maldidier 1993, p. 114). Ainsi lors d'un acte de nomination, dans une construction syntaxique, dans des éditoriaux ou des textes de commentaire, surgissent des bribes de discours autre, qui font partie des mémoires collectives (Halbwachs) sans être forcément partagées par l'ensemble des destinataires de ces textes et sans que soient non plus partagés par les médiateurs et leurs lecteurs les « éclairages » (Grize) qu'ils donnent à l'ordre du texte :

10. [...] la leçon de la crise de la vache folle - on ne joue pas impunément avec la nature - n'a pas encore été tirée par l'Union européenne. [...] Mais le « marché » fait pression, et comme hier pour la vache folle, il encourage l'Union à s'ouvrir à ces nouvelles technologies, source potentielle de profit [...]

11. Ce qu'on appelle manipulation - terme piégé qui disqualifie les nouvelles technologies avant tout débat - en des temps plus optimistes s'appelait tout simplement progrès. Les scientifiques et les ingénieurs agricoles contredisent la nature ? C'est la chose au monde la plus... naturelle.

12. Dans cette nouvelle boîte de Pandore, on peut trouver aussi bien une corne d'abondance (le plus vieux rêve de l'humanité : une nourriture saine, goûteuse et bon marché pour tous) que de possibles fléaux (Frankenstein s'est déguisé en marchand de pop corn).

(Exemples pris dans Libération)

Ces différents niveaux de contextualisation permettent de repérer les différentes communautés convoquées et les genres d'origine des propos rapportés (au fil du texte, il est parfois précisé qu'il s'agit d'extraits d'un rapport, d'une étude, d'un article, d'une interview), lorsqu'ils sont «situés ». Ils incitent en revanche, lorsqu'ils ne le sont pas, ou lorsqu'ils surgissent dans la mémoire d'un mot ou le pré-construit d'une construction syntaxique (Ex. 3, 4, 5, par exemple et au fil des autres), à faire un effort volontaire de mémoire, à rechercher dans les archives et sur l'internet, par exemple, l'origine d'une expression ou d'une prise de position ou d'un point de vue. Ils permettent ainsi de constituer des corpus complémentaires, des corpus de référence qui viennent compléter les corpus exploratoires et les premiers corpus de travail, afin de retrouver les contextes des mots et des dires et de pister leur traçabilité à travers l'histoire et la mémoire, les moments, les communautés, les événements qu'ils ont traversés. Ils exemplifient la conception de l'énonciation du Cercle de Bakhtine: «toute énonciation, quelque signifiante et complète qu'elle soit par elle-même, ne constitue qu'une fraction d'un courant de communication ininterrompue (touchant à la vie quotidienne, la littérature, la connaissance, la politique, etc.). Mais cette communication verbale ininterrompue ne constitue à son tour qu'un élément de l'évolution tous azimuts et ininterrompue d'un groupe social donné » (B/V, 1977, p. 136). 
Sophie Moirand

Cette contextualisation « large » des points d'hétérogénéité énonciative repérées au fil des unités empiriques analysées permet non seulement de caractériser les genres selon leur texture énonciative, mais également selon la fonction que les différentes formes de dialogisme jouent dans l'orientation pragmatique ou argumentative de l'article ou du titre, ce qui fait également partie de l'objectif de l'analyse.

\subsection{La visée de l'analyse}

La relecture des textes du Cercle de Bakhtine, menée conjointement à leur mise à l'épreuve de données empiriques, m'a permis de mieux préciser la visée de l'analyse proposée des genres de la presse quotidienne.

Seul le cadre dialogique m'a paru s'inscrire d'emblée dans une perspective discursive, à la différence des autres théories énonciatives, parce que, comme le souligne Todorov (1981), il met l'intertextualité au centre du schéma de la communication et qu'il donne à la situation une épaisseur historique et sociale, ce que les cadres indiciel et pragmatique ne font pas (Moirand 2005b). Le cadre dialogique place l'énonciation au centre de la constitution des genres et dans ses relations avec les discours antérieurs et à venir d'un événement.

Ce ne sont donc pas les relations interpersonnelles entre les scripteurs et leurs destinataires, ni entre les acteurs autorisés à prendre la parole dans les médias, ni entre ces acteurs et les médiateurs qui m'intéressent. Ce que je cherche à décrire, ce sont les relations interdiscursives entre les discours qui se croisent, se rencontrent sans forcément l'avoir voulu, s’ignorent, se côtoient ou s'interpénètrent, au fil des genres et de leurs rencontres dans la matérialité du support, dans l'espace du numéro et dans la chronologie des événements. Il s'agit donc de penser l'énonciation dans son articulation avec une sémantique discursive, qui tienne compte du sens des mots et des constructions dans leur contexte, c'est-à-dire avec ce qu'ils inscrivent en eux-mêmes de discours autres : les mots et les énoncés ont une histoire, l'objet dont on parle a été pensé avant par d'autres et les noms qu’on lui donne sont toujours « habités » des sens qu'ils ont déjà rencontrés.

Dans cette perspective, on pose que toute actualisation d'un genre discursif a une histoire en même temps qu'il est lui-même dans l'histoire. Ainsi l'interview dialoguée dans la presse écrite s'est développée après que les lecteurs ont intériorisé le genre à la radio : la forme qu'il prend dans la presse est cependant fort éloignée de l'entretien oral qui l'a précédé (il s'agit rarement d'une transcription et les contraintes d'espace et de lisibilité sont importantes). Le développement des médias oraux et du temps d'exposition auquel sont soumis les citoyens des pays développés explique leur influence sur l'évolution des genres de la presse : la rubrique Voix express du Parisien, dans laquelle on interroge quatre à cinq citoyens ordinaires, semble s'inspirer des «micro-trottoirs » de la télévision. Le mélange des genres qui font appel à l'émotion (les photos, les interviews, les témoignages) et des genres qui font appel au cognitif (les croquis, les schémas, les glossaires et les articles d'information) dans le traitement des catastrophes naturelles (le tsunami de 2004, par exemple) semblent proches parfois du traitement télévisé. L'éclatement des informations en micro-genres dans une hyperstructure qui occupe la limite d'une page ou une double page (notion proposée 
par Grosse et Seibold 1996 et reprise dans Lugrin 2001, par exemple) oblige à s'interroger sur l'évolution des genres de la presse et, ce qui nous intéresse désormais davantage, sur le rôle joué par les interactions discursives dans l'orientation pragmatique du genre (qu'on ne fera ici qu'effleurer: voir Moirand 2007a, chap. 4 et 2007b).

On a évoqué plus haut la visée explicative des genres de l'information dans le traitement des événements scientifiques ou technologiques à caractère politique ou social. Un modèle dialogique de l'explication permet d'observer comment alternent citations de spécialistes et explications didactiques du journaliste qui ne dit pas la source de ses connaissances; ainsi, au fil du discours, des paroles savantes s'entremêlent aux dires du journaliste, de telle façon que c'est le journaliste qui joue le rôle de celui qui explique, le spécialiste cautionnant le genre par sa seule « présence » et la présence des quelques dires rapportés au fil du texte, accompagné d'une carte et de croquis (infographie dont on ne connait pas l'origine) :

13. Un tremblement de terre de magnitude 7,6 sur l'échelle de Richter a principalement touché le nord-est du Pakistan (Cachemire) ainsi que le nord de l'Inde (Jammu-Cachemire), l'est de l'Afghanistan et l'ouest de la Chine. L'épicentre est situé [...]. Plus précisément, l'épicentre, c'est-à-dire la zone de la surface terrestre située au-dessus du foyer souterrain, où ont été ressentis les plus importants ébranlements, a été " localisé à une centaine de kilomètres à l'est de $[\ldots]$ », selon les sismomètres très précis du Réseau national de surveillance sismique (Renass) basés au sein de l'Observatoire des sciences de la terre (CNRS-INSU) à Strasbourg. Le séisme s'est produit à 3h50 GMT (8h50 heure locale).

«Sa puissance dévastratrice, c'est-à-dire son intensité, dépend essentiellement de la qualité des constructions et ne peut-être évaluée que par des spécialistes sur le terrain » précise Michel Granet, directeur du réseau.

La catastrophe est provoquée par la «collision» de deux continents, le souscontinent indien et la plaque eurasienne. "La plaque indienne remonte vers le nord à raison de $2 \mathrm{~cm}$ par an, en provoquant de séismes dramatiques [...]", explique Henri Gassier, sismologue à l'Observatoire de Strasbourg. Le mouvement vers le nord du continent indien se poursuit depuis 45 à 50 millions d'années, et est à l'origine des montagnes de la chaîne himalayenne, toujours en formation [...] [La Croix, 10 octobre 2005]

Le même numéro de journal fait paraître un éditorial consacré à cet événement qui part d'un « témoignage » (?) et se termine par une invitation à la raison, adressée à cette région du monde, et justifiée par un certain nombre de rappels d'autres catastrophes et d'appels à l'histoire des relations entre l'homme et la nature :

14. «La nuit dernière, chacun d'entre nous avait une maison et une famille. Maintenant, la plupart d'entre nous sont sans domicile et beaucoup n'ont plus de parents ». Ce témoignage s'est répété à l'infini sur le parcours du violent tremblement de terre qui s'est produit samedi au Cachemire [...].

Ce drame, dont les morts se comptent par dizaines de milliers, les blessés par 
Sophie Moirand

centaines de milliers, porte la marque de la fatalité.[...] A la différence des catastrophes récentes, le tsunami en Asie du Sud, Katrina aux Etats-Unis ou même les dramatiques inondations en Amérique centrale, il n'y a pas lieu, ici, d'interroger les rapports de l'homme et de la nature et les dégradations qu'on lui inflige, ou au moins pas aussi directement...

Tous ces enchaînements d'événements «naturels» meurtriers soulignent cependant par leur accumulation une fragilité oubliée par toutes les sociétés dans leur rapport à la nature. Ces catastrophes à répétition devraient ouvrir des failles dans nos ambitions de maîtrise du monde [...]

Puis viendra peut-être un temps pour la raison. L'Inde et le Pakistan, toutes deux puissances nucléaires, à cause de leur rivalité dans cette partie du monde, seront peut-être amenés à accélérer leur rapprochement amorcé... [...] A tous ceux qui se posent en amis de l'Inde et du Pakistan de les aider à le comprendre. Au Cachemire au moins...

[La Croix, 10 octobre 2005]

Quant à la construction d'un intertexte plurilogal, elle me semble exemplaire de ces rencontres intertextuelles et interdiscursives, caractéristiques d'une évolution récente de certains genres de l'information qui rendent compte de controverses:

15. "TERRORISTES », "obscurantisme », "démarche totalitaire ». Les mots n'étaient pas assez durs, sous la plume du directeur général du groupe Limagrain, pour condamner les destructions de trois parcelles de maïs transgénique, la semaine dernière, dans le sud de la Drôme (le Monde du 16 août). «La coupe est pleine ", a renchéri, mercredi 22 août, un porte-parole du semencier français, au lendemain de nouvelles destructions dans le Gard, qui visaient des parcelles exploitées par l'américain Monsanto. Limagrain s'est dit solidaire de son grand concurrent "quand il s'agit de défendre des activités de recherche ». [...]

La destruction, dans la nuit du 10 au 11 août, des essais de Meristem Therapeutics, une start-up de Clermond-Ferrand qui, depuis une décennie, s'ingénie à transformer des plantes en usines à médicaments (Le Monde du 17 avril), a provoqué un électrochoc. La parcelle arrachée était plantée d'un maïs transformé pour produire de la lipase, utile dans le traitement de la mucoviscidose. "Tout comme il n'y a pas de "bon" nucléaire, l'alibi thérapeutique ne justifie pas plus la poursuite d'un tel processus que les "nécrotechnologies" agroproductivistes», avait expliqué l'organisation qui a revendiqué l'opération. "En s'attaquant sciemment aux OGM destinés à lutter contre les maladies, les opposants montrent jusqu'où ils peuvent aller: il n'y a plus de limites!», estime François Thiboust, directeur des relations extérieures du groupe Aventis CropScience France, et porte-parole des professionnels de la semence et de la protection des plantes [...].

[le Monde, 25 août 2001]

On peut observer comment progresse le texte, alternant la voix des uns et la voix des autres, et la description des actes ou des actes de paroles des acteurs de la controverse. Celle-ci est ainsi «donnée à voir », à travers les désignations des actes et des acteurs figurant dans les paroles rapportées, par exemple les mots obscurantisme et démarche totalitaire dans lesquels sont inscrits des moments particuliers de l'histoire 
ancienne et à travers cette opposition entre deux formations discursives : l'une qui croit à la nature qu'il ne faut pas contrarier et l'autre qui croit qu'il faut la dompter, au nom du progrès. Ainsi chacun des segments entre guillemets renvoie à un texte situé mais également à d'autres discours « clandestins » qui s'inscrivent dans les mots et les dires rapportés. On a pu également observer que les désignations de cette controverse (guerre, bataille mais également fronde et croisade) portaient en elles-mêmes une histoire, qui participe à l'orientation pragmatique ou argumentative du genre. Mais pour cerner l'évolution des genres de la presse à travers les différents types d'interactions discursives qu'ils mettent en jeu, il faut envisager un travail d'observation qui porte sur une période suffisamment longue, ce qui reste à entreprendre.

Si les conceptions du Cercle de Bakhtine nous ont paru apporter des notions opératoires fondamentales à l'analyse des genres de la presse, c'est par le lien qu'il faisait entre les formes de l'énoncé et une réflexion sur l'interaction verbale qui va bien au-delà d'une conception étroite du contexte et de la situation. Car, comme le rappelle J. Peytard (1995 : 36) :

dire “interaction verbale”, ce n'est pas seulement prendre en compte ce qui, dans le face-à-face d'un individu et d'un autre individu, dans un dialogue, psychologiquement, logiquement et linguistiquement, se produit par concaténation, c'est, prioritairement, penser l'interaction réalisée dans/par l'ensemble des multiples discours d'une société donnée, en un moment donné. Penser que ces discours, dans leur multitude indéfinie, interagissent les uns avec les autres. Et tout duo/dialogue singulier ne peut être analysé hors de l'interaction sociodiscursive.

Dans cette perspective, les genres de la presse ne sont donc pas à rapporter à priori à une situation de communication « hic et nunc» (dont Rastier 2007 décrit les limites), qui ne prendrait en compte que les interactions verbales entre les scripteurs des textes et leurs lecteurs potentiels à l'intérieur de l'institution qui les produit. Ils semblent en effet se caractériser, par rapport aux autres genres de l'environnement quotidien, par une particularité qui leur est constitutive: celle d'être des lieux de rencontre entre des discours multiples, appartenant à des communautés langagières différentes et à des époques historiques différentes. Lieux privilégiés d'interactions entre des dires venant de mondes sociaux différents, et parfois d'autres langues et d'autres cultures, ils se caractérisent par les différentes formes de dialogisme qu'ils actualisent et la façon de les distribuer, différemment selon les genres, dans les unités discursives empiriques qu'on rencontre sur l'aire de la page et au fil des numéros. Mais cette distribution, on l'a entrevu, est étroitement dépendante des fonctions qui sont attribuées au genre par les pratiques langagières des communautés professionnelles médiatiques, pratiques qui semblent évoluer au fil du temps, et selon l'orientation pragmatique qui est donnée au texte : information, explication didactique, conseil ou bien encore une explication médiatique proche de l'argumentation (Moirand 2007a, chap. 4 et 2007b). Ce qui permet de reconstruire à posteriori la situation mais une situation au sens de Bakhtine, comme on a tenté de l'expliquer brièvement dans cet article. 


\section{RÉFÉRENCES BIBLIOGRAPHIQUES}

ADAM, J-M. (1997), «Unités rédactionnelles et genres discursifs: cadre général pour une approche de la presse écrite », Pratiques, n 97, pp. 3-18.

Authier-Revuz, J. (1982), "Hétérogénéité montrée et hétérogénéité énonciative : éléments pour une approche de l'autre dans le discours", DRLAV, revue de linguistique, ${ }^{\circ} 26$, pp. 91-151.

Authier-Revuz, J. (2000), «Aux risques de l'allusion », dans Murat, M. (éd.), L'allusion dans la littérature, Paris, Presses universitaires de Paris Sorbonne, pp. 209-235.

BAKHTine, M. (v.n. Volochinov) (1977, traduction [1929]), Le marxisme et la philosophie du langage, Paris, Éditions de Minuit.

BAKhtine, M. (1984, traductions), Esthétique de la création verbale, Paris, Gallimard.

Courtine, J.-J. (éd) (1981), Analyse du discours politique, Langages, $\mathrm{n}^{\circ} 62$.

Cusin-Berche, F. (2000) (éd.), Rencontres discursives entre sciences et politique dans les médias, Les Carnets du CEDISCOR, $\mathrm{n}^{\circ} 6$.

DIJK van, T.A. (2006), « Politique, idéologie et discours », SEMEN, n 21, pp. 73-102.

Grize, J.-B. (1992), «Éclairage » in Grize, J.-B, Un signe parmi d'autres, Hauterive, Suisse, Éditions Gilles Attinger, pp. 22-25.

Grize, J.-B. (2005), «Le point de vue de la logique naturelle» in Doury, M. et Moirand, S. (éds), L'argumentation aujourd'bui. Positions théoriques en confrontation, Paris, Presses Sorbonne Nouvelle, pp. 35-43.

Grosse, E.U. et SEIBOlD, E, (1996), Panorama de la presse parisienne: histoire et actualité, genres et langages, Francfort s/Maine, Peter Lang.

Halbwachs, M. (1994 [1925]), Les cadres sociaux de la mémoire, Paris, Albin Michel.

Halbiwachs, M. (1997 [1950]), La mémoire collective, Paris, Albin Michel.

LugRIN, G. (2001), « Le mélange des genres dans l'hyperstructure », SEMEN n 13, pp. 65-95.

Maldidier, D. (1990), L'inquiétude du discours, Textes de Michel Pêcheux choisis et présentés, Paris, Éditions des Cendres.

MALDIDIER, D. (1993), «L'inquiétude du discours. Un trajet dans l'histoire de l'analyse du discours : le travail de Michel Pêcheux », dans SEMEN, no 9, pp. 107-119.

Micheli, R. (2006), «Contexte et contextualisation en analyse du discours : regard sur les travaux de T. van Dijk », dans SEMEN 21, pp. 103-120.

MoIRAND, S. (1988), Une histoire de discours. Une analyse des discours de la revue Le français dans le monde 1961-1981, Paris, Hachette.

MoIRAND, S. (2000), «Les indices dialogiques de contextualisation dans la presse ordinaire» dans Cabiers de praxématique 33, Sémantique de l'intertexte, pp. 145-184. 
MoIRAND, S. (2001), « Du traitement différent de l'intertexte selon les genres convoqués dans le traitement des événements scientifiques à caractère politique ", SEMEN, $\mathrm{n}^{\circ} 13$, pp. 97-117.

MoIRAND, S. (2003a), «Communicative and Cognitive Dimensions of Discourse on Science in the French Mass Media », Discourse Studies, n 5-2, pp. 175-206.

MoIRAND, S. (2003b), «Quelles catégories descriptives pour la mise au jour de genres du discours?", Actes de la journée scientifique sur Les genres de l'oral, édités en octobre 2003 sur le site de l'UMR ICAR, Lyon 2 et ENS LSH :

http://gric.univ-lyon2.fr/Equipe1/actes/journees_genre.htm

MoIRAND, S. (2004a), «Le texte et ses contextes», dans Adam, J.-M., Grize, J.-B. et Ali Bouacha, A. (éds), Texte et discours: catégories pour l'analyse, Éditions universitaires de Dijon, pp. 129-143.

MOIRAND, S. (2004b), «Nomination, caractérisation et objet de discours : questionnements autour du dialogisme et de la mémoire des mots », dans Cassanas, A., Demange A., Laurent B. et Leclerc, A. (éds), Dialogisme et nomination, Publications de l'université Paul Valéry - Montpellier 3, pp. 27-64.

MOIRAND, S (2005a), «De la médiation à la médiatisation des faits scientifiques et technologiques : où en est l'analyse du discours ?", Actes du colloque Sciences, médias, société, publiés sur le site de l'ENS LSH de Lyon, avril 2005 :

http://sciences-medias.ens-lsh.fr/article.php3 ?id_article=59.

MOIRAND, S (2005b), «Le dialogisme, entre problématiques énonciatives et théories discursives », dans Cabiers de praxématique 43, Aspects du dialogisme, pp. 189-220.

MoIRAND, S. (2006a), «Entre discours et mémoire: le dialogisme à l'épreuve de la presse ordinaire ", TRANEL n ${ }^{\circ} 44$, Intertextualité et interdiscursivité dans les médias, pp. 3955.

MOIRAND, S. (2006b), «De l'aire de la page à l'hyperstructure et à l'écran : comment lire et analyser la presse quotidienne française », CAUCE, Revista international de Filología y su Didáctica, $\mathrm{n}^{\circ}$ 29, Universidad de Sevilla, Espagne, pp. 295-320.

MoIRAND, S. (2007a), Les discours de la presse quotidienne. Observer, analyser, comprendre, Paris, Presses Universitaires de France.

MoIRAND, S. (2007b), «Discours, mémoires et contextes : à propos du fonctionnement de l'allusion dans la presse ", revue en ligne CORELA: http://edel.univ-poitiers.fr/corela/document.php?id=1636

Paveau, M.-A. (2006), Les prédiscours. Sens, mémoire, cognition, Paris, Presses Sorbonne Nouvelle.

PÊCHeux, M. (éd) (1975), Analyse du discours, langue et idéologies, Langages 37.

RASTIER, F. (1998), «Le problème épistémologique du contexte et le statut de l'interprétation dans les sciences du langage », Langages $n^{\circ} 129$, pp. 97-111.

RASTIER, F. (2007), «Communication, interprétation, transmission », SEMEN n 23, pp. 121 138.

Todorov, T (1981), Mikhaïl Bakbtine, le principe dialogique suivi des Ecrits du cercle de Bakbtine, Paris, Seuil. 
Sophie Moirand

Voloshinov, V.N. (1981 [1926]), «Le discours dans la vie et le discours dans la poésie », dans Todorov, T., Mikhaïl Bakbtine, le principe dialogique, pp. 181-215.

Voloshinov, V.N. (1981 1930]), «La structure de l'énoncé», dans Todorov T., Mikhaïl Bakbtine, le principe dialogique, pp. 287-314. 